\title{
Mortalidad atribuida a diabetes mellitus registrada en el Ministerio de Salud de Perú, 2005-2014
}

\author{
Noé Atamari-Anahui, ${ }^{1}$ Maycol Suker Ccorahua-Rios, ${ }^{1}$ Alvaro Taype-Rondan ${ }^{2}$ y \\ Christian R. Mejia ${ }^{3}$
}

Forma de citar

Atamari-Anahui N, Ccorahua-Rios MS, Taype-Rondan A, Mejia CR. Mortalidad atribuida a diabetes mellitus registrada en el Ministerio de Salud de Perú, 2005-2014. Rev Panam Salud Publica. 2018;42:e50. https://doi.org/10.26633/RPSP.2018.50

RESUMEN Objetivo. Estimar la mortalidad atribuida a diabetes mellitus (DM) registrada en el Ministerio de Salud (MINSA) de Perú y su asociación con el índice de desarrollo humano $(\mathrm{IDH})$.

Métodos. Estudio ecológico basado en un análisis secundario de los registros de defunción del MINSA de 2005 a 2014. Se consideró mortalidad atribuida a DM a aquellos registros de defunción que tuvieron como causa básica de muerte a la DM. Se presentó la mortalidad atribuida a DM de forma descriptiva y con análisis geoespaciales, y se evaluó la asociación entre la diferencia de la mortalidad asociada a DM (entre 2005-2006 y 2013-2014) y el IDH en los departamentos del Perú mediante la Rho de Spearman.

Resultados. En los 10 años evaluados, se observaron 25074 registros que tuvieron como causa básica del fallecimiento la DM. La mortalidad atribuida a DM por cada 100 mil habitantes aumentó de 5,7 en 2005 a 9,5 en 2014. La mortalidad atribuida a DM representó 2,7\% de los fallecimientos registrados para el periodo evaluado: 3,5\% en la costa, 1,4\% en la sierra y $2,5 \%$ en la selva. Se encontró una asociación directa entre el IDH y la diferencia de mortalidad atribuida a DM (Rho de Spearman $=0,41 ; p=0,04$ ).

Conclusiones. La mortalidad atribuida a la DM ha aumentado en el periodo estudiado, y fue más alta en la costa, intermedia en la selva y menor en la sierra. Se encontró una asociación directa entre el IDH y la diferencia de mortalidad atribuida a la DM.

Palabras clave Diabetes mellitus; mortalidad; Perú.

Actualmente se considera que la diabetes mellitus (DM) es uno de los mayores problemas de salud pública en el mundo por su alta prevalencia y gran impacto en la calidad de vida de las personas que la padecen (1). Se estimó que en 2015 la prevalencia de DM en los

\footnotetext{
Escuela de Medicina, Universidad Nacional de San Antonio Abad del Cusco, Cusco, Perú. Enviar la correspondencia a Noé Atamari-Anahui, noe. atamari@gmail.com
}

adultos de 20 a 79 años de edad ascendía a $8,8 \%$ en el mundo y a $9,4 \%$ en los países de la Región de las Américas, cifras que se encuentran en constante aumento (1). En Perú, la prevalencia estimada para 2012 fue $7 \%$ entre los adultos mayores de 25 años (2).

\footnotetext{
Universidad San Ignacio de Loyola, Lima Perú.

3 Coordinación de Investigación, Universidad Continental, Huancayo, Perú.
}

La DM desencadena complicaciones que disminuyen la esperanza de vida de los pacientes (3), sobre todo en quienes no mantienen un control médico adecuado de la enfermedad. Además, es una de las causas principales de mortalidad a nivel mundial $(4,5)$. Según la Federación Internacional de Diabetes, en 2015 fallecieron por DM cinco millones de personas en todo el mundo (1) y para Perú, la Organización Mundial de la Salud

Este es un artículo de acceso abierto distribuido bajo los términos de la licencia Creative Commons Attribution-NonCommercial-NoDerivs 3.0 IGO, que permite su uso, distribución y reproducción en cualquier medio, siempre que el trabajo original se cite de la manera adecuada. No se permiten modificaciones a los artículos ni su uso comercial. Al reproducir un artículo no debe haber ningún indicio de que la OPS o el artículo avalan a una organizaciőn un producto específico. El uso del logo de la OPS no está permitido. Esta leyenda debe conservarse, junto con la URL original del artículo. 
(OMS) ha notificado una mortalidad atribuida a esta enfermedad de $2 \%$ en la población general (6).

El Índice de Desarrollo Humano (IDH) es un indicador que permite comparar el desarrollo entre países o regiones, y consta de tres componentes: educación, salud y producto interior bruto (7). Aunque estos factores pueden tener efectos positivos en la salud en general, la urbanización y la industrialización asociadas con el IDH pueden influir en ciertos factores de riesgo para la salud. Se ha comprobado que las regiones con mayor IDH tienen frecuencias más elevadas de factores de riesgo de enfermedades cardiometabólicas, como la inactividad física (8) y la dieta hipercalórica (9), que contribuyen al desarrollo de DM (10). El IDH también se ha asociado directamente con la morbimortalidad por DM $(9,11)$, y a nivel mundial parece explicar $8,6 \%$ de la varianza de la prevalencia de DM, aunque esta relación pueda estar sujeta a variaciones por países y regiones (12).

En Perú no se han encontrado informes que describan la mortalidad atribuida a la DM, su evolución temporal, su distribución geográfica, ni su asociación con el IDH. Esta información sería útil para dirigir intervenciones e incluso para distribuir mejor los recursos asignados para la prevención de la mortalidad por DM en el país $(1,13)$. Por ello, el objetivo del presente estudio fue describir la mortalidad atribuida a DM registrada en el Ministerio de Salud del Perú (MINSA) durante el periodo 2005-2014 y su asociación con el IDH.

\section{MATERIALES Y MÉTODOS}

Se realizó un estudio ecológico con datos secundarios y anonimizados obtenidos de una fuente de información pública. La unidad de análisis fue cada uno de los 25 departamentos del Perú. Se solicitó la base de datos de los certificados de defunción emitidos por el MINSA (con variables agrupadas por cada departamento del Perú) a través la Plataforma de Acceso a la Información Pública del MINSA (http:/ / www.minsa.gob.pe/portada/transparencia/solicitud/frmFormulario.asp).

En Perú, cuando una persona fallece, debe elaborarse un certificado de defunción para poder evaluar las estadísticas demográficas, epidemiológicas y médico-legales sobre la mortalidad (14). Este certificado de defunción puede ser emitido por el MINSA, EsSalud, la policía o por mandato judicial. Todos los certificados son recolectados por el Registro Nacional de Identificación y Estado Civil (RENIEC) (15).

La mayor parte de estos certificados de defunción son emitidos por el MINSA, que presta servicios a más de la mitad de la población con seguro de salud en el Perú (16). Estos certificados son rellenados por el profesional de la salud que certifica el deceso de una persona en cada jurisdicción de las Direcciones Regionales de Salud o Direcciones de Salud o las que hagan las veces de ellas bajo la Resolución Ministerial No. 826-2005 del MINSA (17).

En el certificado de defunción se registran tres tipos de causas de muerte: directa, intermedia, y básica. Para realizar el análisis, se tuvo en cuenta la causa de muerte básica, definida como la enfermedad que inicia la cadena de acontecimientos patológicos que conducen directamente a la muerte (18). Esta causa es utilizada por el MINSA para elaborar estadísticas de mortalidad atribuida a alguna enfermedad, ya que las causas de muerte se codifican con la Clasificación Internacional de Enfermedades (CIE 10).

La variable de interés del estudio fue la frecuencia de muertes atribuidas a la DM. Esta variable se obtuvo de la base de datos de los certificados de defunción emitidos por el MINSA. Para determinar las muertes atribuidas a DM se consideraron los certificados que presentaron como causa de muerte básica los códigos correspondientes a DM: del E10 al E14. Esta variable se evaluó por año y por departamento dividiendo el número anual de muertes atribuidas a la DM por el número de habitantes de cada departamento en cada año evaluado. Para ello, el número de habitantes de cada departamento y para cada uno de los años de estudio se obtuvo de la página web del Instituto Nacional de Estadística e Informática del Perú (http:// proyectos.inei.gob.pe/web/poblacion/).

Las otras variables de los fallecidos (la edad, el sexo y el departamento donde se registró el fallecimiento) que se utilizaron en este estudio también se obtuvieron de la base de datos de los certificados de defunción emitidos por el MINSA. Además, se utilizaron las siguientes variables de los departamentos donde se registró el fallecimiento: su región natural geográfica (costa, sierra, o selva, según la distribución del Instituto Nacional de Estadística e Informática del Perú) (19) y su IDH para 2012 (obtenido del portal web del Programa de las
Naciones Unidas para el Desarrollo: http: / / www.pe.undp.org / content/ peru/es/home/library/poverty/Informesobredesarrollohumano2013/IDHPeru2013.html).

Para realizar el análisis descriptivo, se calcularon las frecuencias absolutas y relativas de las variables estudiadas. Asimismo, se realizó el análisis exploratorio de los datos espaciales de cada departamento a partir de la integración de la mortalidad atribuida a la DM en la hoja de cálculo de Microsoft Excel® sobre la base de la cartografía obtenida del portal web del Ministerio del Ambiente del Perú. Con este fin, se dividió a los departamentos por quintiles de frecuencia de mortalidad atribuida a la DM. Para realizar estos cálculos se utilizó el programa QGIS v2.10.1 (OSGeo, Beaverton, OR, USA).

Para analizar la asociación entre la diferencia de frecuencias de mortalidad atribuida a la DM entre 2005-2006 y 2013-2014 y el IDH, se utilizó el coeficiente de correlación Rho de Spearman. Se decidió promediar las frecuencias de mortalidad de los dos primeros años evaluados (2005 y 2006) y de los dos últimos (2013-2014) para evitar imprecisiones por tomar un solo año como referencia.

\section{RESULTADOS}

En el periodo 2005-2014, 25074 personas se registraron en la base de datos del MINSA como fallecidas por DM como causa básica, de las cuales 51,8\% fueron mujeres y el 78,6\% tenían 60 o más años de edad.

La mortalidad atribuida a DM representó $2,7 \%$ de los fallecimientos registrados en el periodo 2005-2014 (2,1\% en 2005 y 3,9\% en 2014). La mortalidad atribuida a la DM fue más alta en la costa $(3,5 \%)$, intermedia en la selva $(2,5 \%)$ y menor en la sierra $(1,4 \%)$ (cuadro 1$)$. La tasa de mortalidad general fue 5,7 en el 2005 y 9,5 en el 2014. Hubo un incremento en las tres regiones, con predominio de la costa respecto a las media general (figura 1).

Al evaluar la diferencia de la mortalidad atribuida a la DM (por 100000 habitantes) entre el periodo 2005-2006 y el periodo 2013-2014, los departamentos con mayor incremento de mortalidad atribuida a DM en la costa fueron Moquegua $(12,5)$, Lambayeque $(9,5)$ y Piura $(8,9)$; en la sierra, Puno $(3,7)$, Junín $(3,5)$ y Arequipa $(2,8)$, y en la selva, Madre de 
CUADRO 1. Mortalidad atribuida a la diabetes mellitus (DM) registrada en el Ministerio de Salud de Perú (MINSA) según la región natural y el año del registro de la defunción del periodo 2005-2014

\begin{tabular}{|c|c|c|c|c|c|c|c|c|c|}
\hline \multicolumn{10}{|c|}{ Número de defunciones por región } \\
\hline \multirow[t]{2}{*}{ Año } & \multicolumn{2}{|c|}{ Costa } & \multicolumn{2}{|c|}{ Sierra } & \multicolumn{2}{|c|}{ Selva } & \multicolumn{2}{|c|}{$\begin{array}{l}\text { Total de defunciones en } \\
\text { pacientes con DM en el Perú }\end{array}$} & \multirow{2}{*}{$\begin{array}{c}\text { Total de defunciones registradas } \\
\text { por todas las causas } \\
\text { No. }\end{array}$} \\
\hline & No. & $(\%)^{*}$ & No. & $(\%)^{\star}$ & No. & $(\%)^{*}$ & No. & $(\%)^{\star *}$ & \\
\hline 2005 & 1441 & 2,9 & 310 & 0,9 & 76 & 1,6 & 1827 & 2,1 & 88704 \\
\hline 2006 & 1299 & 3,0 & 400 & 1,1 & 104 & 2,1 & 1803 & 2,2 & 82620 \\
\hline 2007 & 1598 & 3,2 & 414 & 1,2 & 105 & 2,3 & 2117 & 2,4 & 87496 \\
\hline 2008 & 1461 & 2,7 & 397 & 1,2 & 104 & 2,0 & 1962 & 2,1 & 91290 \\
\hline 2009 & 1784 & 3,1 & 382 & 1,1 & 93 & 1,8 & 2259 & 2,4 & 95722 \\
\hline 2010 & 2095 & 3,5 & 426 & 1,2 & 154 & 2,9 & 2675 & 2,7 & 99334 \\
\hline 2011 & 1909 & 3,2 & 446 & 1,4 & 163 & 3,0 & 2518 & 2,6 & 96852 \\
\hline 2012 & 2207 & 3,7 & 536 & 1,6 & 141 & 2,7 & 2884 & 2,9 & 97951 \\
\hline 2013 & 2557 & 4,3 & 534 & 1,6 & 156 & 3,4 & 3247 & 3,4 & 96228 \\
\hline 2014 & 3014 & 4,9 & 628 & 2,0 & 140 & 3,6 & 3782 & 3,9 & 96460 \\
\hline Total & 19365 & 3,5 & 4473 & 1,4 & 1236 & 2,5 & 25074 & 2,7 & 932657 \\
\hline
\end{tabular}

Fuente: elaboración propia a partir del análisis de la información de la base de datos del MINSA de Perú.

*Porcentaje que resulta de dividir el número de defunciones por el total de defunciones por región/año.

**Porcentaje que resulta de dividir el número de defunciones por el total de defunciones por año.

FIGURA 1. Evolución de las tasas de mortalidad atribuida a DM registradas en el Ministerio de Salud de Perú (MINSA) del periodo 2005-2014

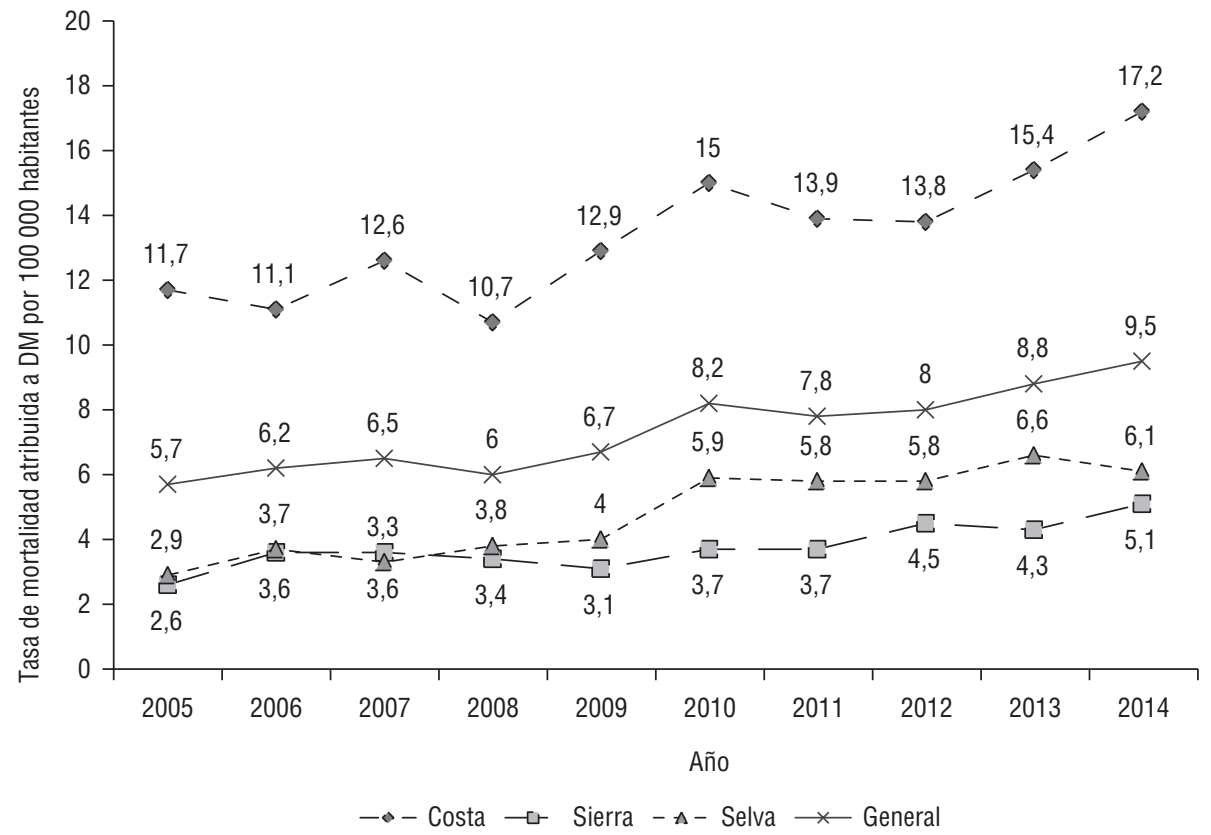

Fuente: elaboración propia a partir del análisis de la información de la base de datos del MINSA de Perú.

Dios $(7,6)$, San Martín $(4,2)$ y Amazonas $(2,9)$. Sin embargo, se observó una disminución en dos departamentos de la costa: Callao $(-6,3)$ y Tumbes $(-6,0)$, en un departamento de la selva, Loreto $(-2,2)$, y en un departamento de la sierra, Cusco $(-1,3)$ (cuadro 2 y figura 2).

La Rho de Spearman $(0,41)$ utilizada para analizar la asociación entre el IDH y la diferencia de tasas de mortalidad (por 100000 habitantes) atribuidas a DM entre 2005-2006 y 2013-2014 por departamento fue estadísticamente significativa $(P=0,04)$ (figura 3).

\section{DISCUSIÓN}

La mayor mortalidad atribuida a la DM y su mayor aumento se detectaron en los departamentos de la costa de Perú, lo cual es esperable por la mayor prevalencia de DM estimada para la costa
$(8,2 \%)$ en comparación con la sierra $(4,5 \%)$ y la selva $(3,5 \%)$ (2). Esto podría deberse a que en la costa se encuentran varias de las ciudades más pobladas del país, donde al ser más frecuentes los hábitos de vida urbana, como el sedentarismo, la obesidad y la dieta inadecuada, mayor es la probabilidad de padecer DM $(20,21)$.

Para el periodo 2005-2014, se observó un aumento de la mortalidad en la mayoría de departamentos de Perú, que fue mayor en los de Lambayeque, Moquegua, Piura y La Libertad. Esto puede explicarse por el acelerado desarrollo y urbanización de las principales ciudades de dichos departamentos, que, a su vez, es posible que se haya acompañado de un aumento de la frecuencia de síndrome metabólico, sedentarismo y otros factores de riesgo de la DM (22). Sin embargo, otras ciudades del Perú, de la sierra y la selva están entrando en un periodo de transición epidemiológica y es posible que en ellos en los próximos años aumenten tanto el número de casos de DM como de las tasas de mortalidad (23).

Además, se observó una disminución de la mortalidad en dos departamentos de la costa (Callao y Tumbes), en uno de la sierra (Cusco) y en otro de la selva (Loreto), que podría deberse a la existencia de factores protectores que deben analizarse en futuros estudios o a flujos de emigración de grupos en riesgo desde estos departamentos a zonas de mayor desarrollo socioeconómico en el periodo estudiado (24). 
CUADRO 2. Mortalidad atribuida a la diabetes mellitus registrada en el Ministerio de Salud de Perú (MINSA) por departamentos: comparación de los periodos 2005-2006 y 2013-2014

\begin{tabular}{|c|c|c|c|c|}
\hline \multirow[b]{2}{*}{ Departamento } & \multicolumn{4}{|c|}{ Tasa de mortalidad x 100000 habitantes } \\
\hline & $\begin{array}{c}2005-2006 \\
(\mathrm{t} 1)\end{array}$ & $\begin{array}{c}2013-2014 \\
\text { (t2) }\end{array}$ & $\begin{array}{l}\text { Diferencia de } \\
\text { tasas (t2-t1) }\end{array}$ & $\begin{array}{c}\text { Razón de tasas (\%) } \\
([\mathrm{t} 2 / \mathrm{t} 1] \times 100)\end{array}$ \\
\hline Perú (País) & 6,0 & 9,1 & 3,1 & 149,9 \\
\hline Región Costa & 11,4 & 16,3 & 4,9 & 142,5 \\
\hline Callao & 20,0 & 13,7 & $-6,3$ & 68,7 \\
\hline Ica & 14,8 & 18,4 & 3,6 & 124,4 \\
\hline La Libertad & 8,3 & 17,1 & 8,8 & 205,3 \\
\hline Lambayeque & 11,3 & 20,8 & 9,5 & 183,4 \\
\hline Lima & 6,9 & 15,6 & 8,7 & 226,0 \\
\hline Moquegua & 5,8 & 18,3 & 12,5 & 314,2 \\
\hline Piura & 9,2 & 18,1 & 8,9 & 195,2 \\
\hline Tacna & 13,5 & 17,8 & 4,3 & 131,2 \\
\hline Tumbes & 12,9 & 6,9 & $-6,0$ & 53,2 \\
\hline Región Sierra & 3,1 & 4,7 & 1,6 & 151,7 \\
\hline Ancash & 3,0 & 5,5 & 2,5 & 182,4 \\
\hline Apurímac & 1,8 & 3,1 & 1,3 & 167,1 \\
\hline Arequipa & 9,7 & 12,5 & 2,8 & 128,3 \\
\hline Ayacucho & 1,6 & 2,9 & 1,3 & 177,7 \\
\hline Cajamarca & 2,3 & 4,5 & 2,2 & 193,6 \\
\hline Cusco & 3,3 & 2,0 & $-1,3$ & 60,8 \\
\hline Huancavelica & 1,5 & 1,9 & 0,4 & 126,7 \\
\hline Huánuco & 3,1 & 3,8 & 0,7 & 122,7 \\
\hline Junín & 3,5 & 7,0 & 3,5 & 197,8 \\
\hline Pasco & 1,6 & 2,5 & 0,9 & 156,3 \\
\hline Puno & 2,7 & 6,4 & 3,7 & 233,3 \\
\hline Región Selva & 3,3 & 6,4 & 3,1 & 191,9 \\
\hline Amazonas & 1,4 & 4,3 & 2,9 & 312,5 \\
\hline Loreto & 4,3 & 2,1 & $-2,2$ & 49,3 \\
\hline Madre de Dios & 3,3 & 10,9 & 7,6 & 335,4 \\
\hline San Martín & 2,8 & 7,0 & 4,2 & 250,0 \\
\hline$\underline{\text { Ucayali }}$ & 4,8 & 7,4 & 2,6 & 153,1 \\
\hline
\end{tabular}

Fuente: elaboración propia a partir del análisis de la información de la base de datos del MINSA de Perú.

Al mismo tiempo, el ascenso en los últimos 10 años de la tasa de mortalidad atribuida a la DM (figura 1) podría deberse al aumento del número de casos registrados como consecuencia de la mejora del sistema de información de Perú (25). Por tanto, es probable que en el primer grupo de departamentos se deba hacer hincapié en la prevención secundaria, mientras que en el resto de departamentos el énfasis deba recaer en la prevención primaria de la DM. Asimismo, la mortalidad atribuida a la DM podría variar, incluso dentro de un mismo departamento $y$, por este motivo, el enfoque y las estrategias de prevención deben adecuarse a cada contexto.

En las regiones con mayor IDH la mortalidad por DM fue más elevada, lo que es congruente con los resultados de estudios previos que indican que en las regiones más desarrolladas la prevalencia y la mortalidad por DM son más altas $(2,9,12)$. Estos resultados puede ser tributarios de los cambios en los estilos de vida y de los patrones de la alimentación, dado que la industrialización de los alimentos (26), la inactividad física y la obesidad son más frecuentes en países con mayor IDH (8).

La asociación entre el IDH y la mortalidad por DM sugiere que es necesario que el desarrollo y el crecimiento económico de las regiones se acompañe de una adecuada educación en estilos de vida, del control de los factores de riesgo, así como de una gestión adecuada de sus recursos económicos destinados al control y a la prevención de esta enfermedad (27).
Como principales limitaciones de este estudio cabe mencionar las siguientes. Primero, los datos analizados provienen del registro de fallecimientos notificados al MINSA, no de los de otras instituciones; aunque el MINSA notifica la mayor parte de fallecimientos en Perú, no hay motivos para pensar que la tasas de mortalidad atribuida a la DM basada en las muertes notificadas por otras instituciones sean muy distintas. Segundo, este es un estudio con fuentes secundarias que podría tener deficiencias en la recogida de datos. Además, la notificación de muertes atribuidas a la DM podría no ser óptima al depender de personal de salud con escasa capacitación para rellenar la ficha de defunción. Esto podría alterar las tendencias de muertes atribuidas a la DM, porque la calidad de las bases de datos y la capacitación del personal de salud pueden haber mejorado en años recientes o pueden diferir entre departamentos. Tercero, al evaluar la asociación entre el IDH y la diferencia entre tasas de mortalidad por departamento puede incurrirse en la falacia ecológica y por esta razón no se puede asegurar que esta asociación se dé a nivel de ciudades, barrios o individuos (28). Por último, la base de datos evaluada no permitió realizar estandarizaciones por edad, por lo cual esta variable podría estar confundiendo en alguna medida la asociación entre el IDH y la mortalidad por DM. A pesar de estas limitaciones, este es uno de los primeros estudios que describen la mortalidad atribuida a la DM en Perú y en ella se han desagregado los resultados por años y departamentos y evaluado su asociación con el IDH.

Como conclusión debe decirse que, según los registros del MINSA, la mortalidad atribuida a la DM en Perú aumentó entre 2005 y 2014 y fue mayor en la costa, intermedia en la selva y menor en la sierra. Los departamentos de Moquegua, Lambayeque y Piura fueron los que presentaron mayor aumento de la mortalidad por 100000 habitantes. Además, se encontró una asociación directa entre el IDH y la mortalidad atribuida a la DM. Dado que se trata de un estudio ecológi$\mathrm{CO}$, al interpretar sus resultados debe tenerse en cuenta la posibilidad de incurrir en la falacia ecológica.

Los resultados obtenidos animan a que en futuros estudios se evalúen en profundidad los factores que están determinando el aumento de la mortalidad atribuida a la DM en Perú y 


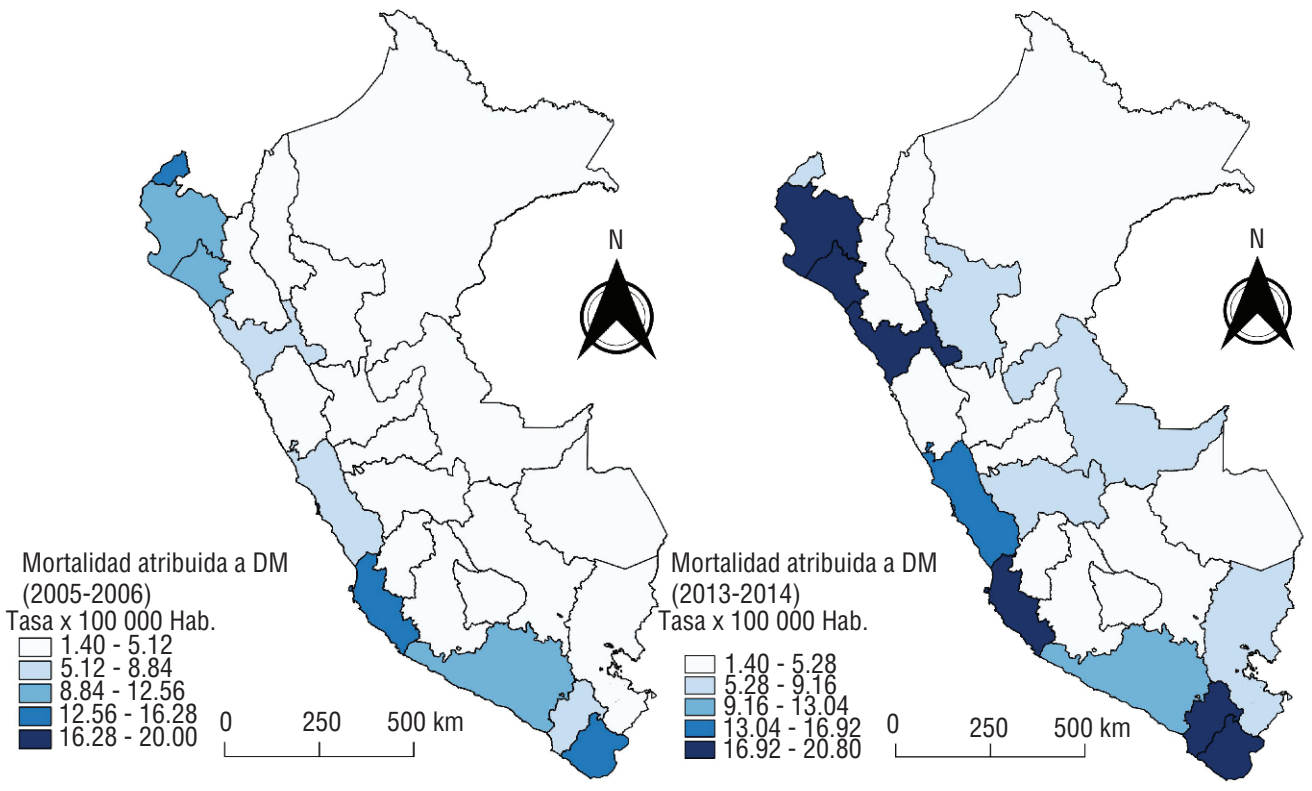

Fuente: elaboración propia a partir del análisis de la información de la base de datos del MINSA de Perú.

FIGURA 3. Asociación entre el Índice de Desarrollo Humano y la diferencia de tasas de mortalidad atribuida a la DM entre el periodo 2005-2006 (t1) y el periodo 2013-2014 (t2)

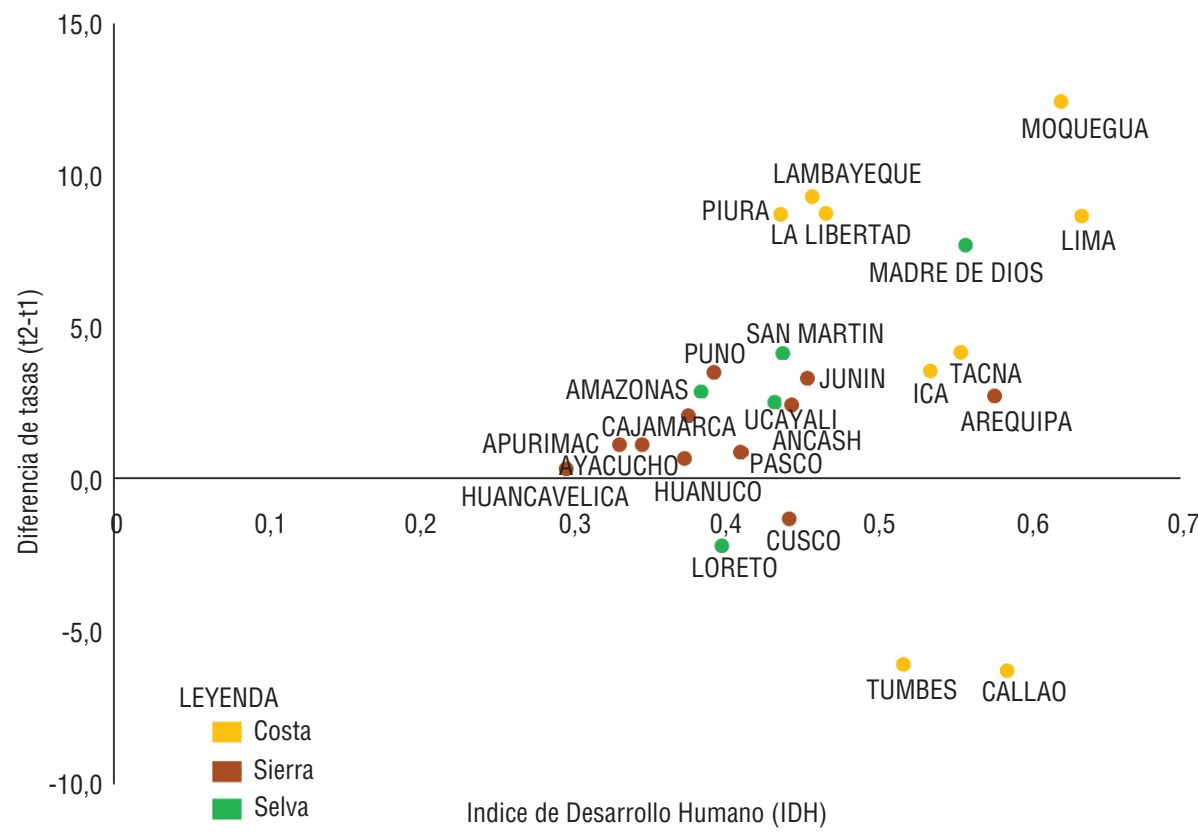

Fuente: elaboración propia a partir del análisis de la información de la base de datos del Ministerio de Salud de Perú.

Nota: Rho de Spearman $=0,413(P=0,04)$. especialmente en los departamentos de la costa. Asimismo, la asociación directa entre IDH y mortalidad por DM plantea la necesidad de que el desarrollo y el crecimiento económico de las regiones se acompañen de una adecuada educación en estilos de vida, factores de riesgo y en la gestión adecuada de los recursos económicos asignados al control y a la prevención de esta enfermedad.

Agradecimientos. Los autores agradecen la colaboración en el diseño de los gráficos de este estudio a Julio César Sucasaca-Rodríguez y Reyner Puma-Quehuarucho.

Financiamiento. Este estudio no recibió ningún tipo de financiación.

Conflictos de interés. Los autores declaran no tener conflictos de interés.

Declaración. Las opiniones expresadas en este manuscrito son responsabilidad de los autores y no reflejan necesariamente los criterios ni la política de la RPSP/ PAJPH y/o de la OPS. 


\section{REFERENCIAS}

1. International Diabetes Federation. Atlas de la diabetes de la FID. 7th ed. Bruselas: IDF; 2015;51. Disponible en: http://www. fundaciondiabetes.org/upload/publicaciones_ficheros/95/IDF_Atlas_2015_SP WEB_oct2016.pdf Acceso el 8 de febrero de 2017.

2. Seclen SN, Rosas ME, Arias AJ, Huayta E, Medina CA. Prevalence of diabetes and impaired fasting glucose in Peru: report from PERUDIAB, a national urban population-based longitudinal study. BMJ Open Diabetes Res Care. 2015;3(1):e000110.

3. Atamari-Anahui N, Martinez-Ninanqui FW, Paucar-Tito L, Morales-Concha L, MirandaChirau A, Gamarra-Contreras MA, et al. Factors associated to inpatient mortality rates in type-2-diabetic patients: a cross-sectional analytical study in three Peruvian hospitals. Medwave. 2017;17(9):e7097.

4. IDF Diabetes Atlas Group. Update of mortality attributable to diabetes for the IDF Diabetes Atlas: Estimates for the year 2013. Diabetes Res Clin Pract. 2015; 109(3):461-5.

5. Agudelo-Botero M, Dávila-Cervantes CA. Carga de la mortalidad por diabetes mellitus en América Latina 2000-2011: los casos de Argentina, Chile, Colombia y México. Gac Sanit. 2015;29(3):172-7.

6. World Health Organization. Diabetes country profiles 2016. Geneva: WHO; 2016. Disponible en: http://www.who. int/diabetes / country-profiles / per_en. pdf?ua=1 Acceso 27 de julio de 2017.

7. Morse S. Stirring the pot. Influence of changes in methodology of the Human Development Index on reporting by the press. Ecol Indic. 2014;45(suppl C):245-54.

8. Atkinson K, Lowe S, Moore S. Human development, occupational structure and physical inactivity among 47 low and middle income countries. Prev Med Rep. 2016;3(suppl C):40-5.

9. Soto-Estrada G, Moreno Altamirano L, García-García JJ, Ochoa Moreno I, Silberman M. Trends in frequency of type 2 diabetes in Mexico and its relationship to dietary patterns and contextual factors. Gac Sanit. 2017. http://dx.doi.org/10. 1016/j.gaceta.2017.08.001

10. Kahn SE, Cooper ME, Del Prato S. Pathophysiology and treatment of type 2 diabetes: perspectives on the past, present, and future. Lancet. 2014;383(9922): 1068-83.

11. Mendoza-Romo MÁ, Zavala-Cruz GG, Padrón-Salas A, Ortiz-Nesme FJ, RamírezArriola MC, Salas-Jiménez JA. Asociación del índice de desarrollo humano y diabetes mellitus tipo 2 en unidades de medicina familiar del estado San Luis Potosí, México. Aten Fam. 2017;24(4):156-9.

12. Mendoza-Romo M, Padrón-Salas A, Cossío-Torres P, Soria-Orozco M. Prevalencia mundial de la diabetes mellitus tipo II y su relación con el índice de desarrollo humano. Rev Panam Salud Publica. 2017;41(e103):1-6.

13. Díaz-Díaz O. Importancia de los estudios de mortalidad en diabéticos. Rev Cubana Endocrinol. 2001;12(3):137-8.

14. Jorge-Miguez AN. La importancia de llenar bien el certificado de defunción. MediSur. 2009;7(6):1-2.

15. Instituto Nacional de Estadistica e Informática. Perú: Natalidad, mortalidad y nupcialidad, 2015. Lima: INEI; 2016. Disponible en: http://www.inei.gob.pe/ media/MenuRecursivo/publicaciones digitales/Est/Lib1407/libro.pdf Acceso el 14 de abril de 2017.

16. Instituto Nacional de Estadistica e Informática. Perú: Sintesis Estadistica 2015. Lima: INEI; 2015:28-9.

17. Oficina General de Estadistica e Informática. Manual para el llenado del formulario de defuncion. Lima: Oficina General de Estadistica e Informática; 2009. Disponible en: http://sisweb.reniec.gob. pe/PortalRegCivil/getFileLeg.htm?hoja=802.pdf Acceso el 14 de abril de 2017.

18. Ministerio de Salud del Perú. Guía técnica para el correcto llenado del certificado de defunción. Lima: MINSA; 2017:30. Disponible en: https:/ /www.saludarequipa.gob.pe/redislay/descargas/RM214 2017MINSA.pdf Acceso el 21 de agosto de 2017.

19. Instituto Nacional de Estadistica e Informática. Distribución de la Población por Departamentos Lima: INEI; 2015. Disponible en: https://www.inei.gob.pe/ media/MenuRecursivo/publicaciones_ digitales/Est/Lib0015/cap-51.htm Acceso el 14 de abril de 2017.
20. Álvarez-Dongo D, Sánchez-Abanto J, Gómez-Guizado G, Tarqui-Mamani C. Sobrepeso y obesidad: prevalencia y determinantes sociales del exceso de peso en la población peruana (2009-2010). Rev Peru Med Exp Salud Publica. 2012; 29(3):303-13.

21. Zafra-Tanaka JH, Millones-Sánchez E, Retuerto-Montalvo MA. Factores sociodemográficos asociados a actividad física y sedentarismo en población peruana adulta. Revista Peruana Epidemiol (en línea). 2013;17(3):1-6.

22. Pajuelo J, Sánchez J. El síndrome metabólico en adultos, en el Perú. An Fac Med. 2007;68(1):38-46.

23. Bernabé-Ortiz A, Carrillo-Larco RM, Gilman RH, Miele CH, Checkley W, Wells JC, et al. Geographical variation in the progression of type 2 diabetes in Peru: The CRONICAS Cohort Study. Diabetes Res Clin Pract. 2016;121(11):135-45.

24. Miranda JJ, Wells JCK, Smeeth L Transiciones en contexto: Hallazgos vinculados a migración rural-urbana y enfermedades no transmisibles en Perú. Rev Peru Med Exp Salud Publica. 2012;29(3):366-72.

25. Curioso WH. eSalud en Perú: implementación de políticas para el fortalecimiento de sistemas de información en salud. Rev Panam Salud Pública. 2014;35(5/6): 437-41.

26. Montonen J, Knekt $P$, Härkänen $T$, Järvinen R, Heliövaara M, Aromaa A, et al. Dietary Patterns and the Incidence of Type 2 Diabetes. Am J Epidemiol. 2005;161(3): 219-27.

27. Taype-Rondan A, Lazo-Porras M, MoscosoPorras M, Moreano-Sáenz M, Miranda JJ. Inadequate glycaemic control in LMIC: health system failures in Peru. Br J Gen Pract. 2016;66(645):197.

28. Borja-Aburto VH. Estudios ecológicos. Salud Pública Méx. 2000;42(6):533-8.

Manuscrito recibido el 27 de agosto de 2017. Aceptado para publicación, tras revisión, el 13 de enero de 2018. 
ABSTRACT

Mortality attributable to diabetes mellitus as recorded by the Ministry of Health of Peru, 2005-2014

Key words Diabetes mellitus; mortality; Peru. index (HDI). attributable to DM.
Objective. To estimate the mortality attributable to diabetes mellitus (DM) as recorded by Peru's Ministry of Health and its association with the human development

Methods. This was an ecological study based on a secondary analysis of death records belonging to the Ministry of Health for the period from 2005 to 2014. A death was considered attributable to DM if the underlying cause of death given in the death record was DM. Mortality attributable to DM has been presented descriptively and in terms of geospatial analyses, and Spearman's rho was used to test for an association between the difference in the mortality attributable to DM (between 2005-2006 and 2013-2014) and the HDI in Peru's various departments.

Results. In the 10-year period under evaluation, 25074 records listed DM as the underlying cause of death. The mortality rate attributable to DM per 100000 population increased from 5.7 in 2005 to 9.5 in 2014 . This accounted for $2.7 \%$ of the deaths recorded during the study period: $3.5 \%$ in coastal areas, $1.4 \%$ in the highlands, and $2.5 \%$ in the rainforest region. A direct association was found between the HDI and the difference in mortality attributable to DM (Spearman's rho $=0.41 ; \mathrm{p}=0.04$ ).

Conclusions. Mortality attributable to DM increased over the study period. It was highest in coastal areas, intermediate in the rainforest region, and lowest in the highlands. A direct association was found between the HDI and the difference in mortality
RESUMO

Mortalidade atribuída à diabetes mellitus nos registros do Ministério da Saúde do Peru, 2005-2014

Palavras-chave
Objetivos. Estimar a mortalidade atribuída à diabetes mellitus com base em registros do Ministério da Saúde do Peru e examinar a associação com o índice de desenvolvimento humano (IDH).

Métodos. Estudo ecológico realizado com dados de uma análise secundária dos registros de óbitos do Ministério da Saúde para o período 2005 a 2014. Foi considerada mortalidade atribuída à diabetes mellitus os registros de óbitos com a diabetes como causa básica de morte. A mortalidade atribuída à diabetes foi apresentada de forma descritiva e com análises geoespaciais e foi feita uma análise com o uso do coeficiente de correlação (rho) de Spearman da associação entre a diferença da mortalidade associada à diabetes (entre 2005-2006 e 2013-2014) e o IDH nas províncias peruanas.

Resultados. No período estudado de 10 anos, foram registados 25.074 óbitos com a diabetes como causa básica de óbito. Observou-se um aumento da mortalidade atribuída à diabetes por 100 mil habitantes, de 5,7 em 2005 a 9,5 em 2014. Ela foi responsável por 2,7\% dos óbitos registrados no período estudado: 3,5\% na região litorânea, 1,4\% na região serrana e $2,5 \%$ na região de floresta. Verificou-se uma associação direta entre o IDH e a diferença de mortalidade atribuída à diabetes (rho de Spearman =0,41; $\mathrm{p}=0,04$ ). Conclusões. A mortalidade atribuída à diabetes aumentou no período estudado, sendo mais elevada na região litorânea, intermediária na região de floresta e mais baixa na região serrana. Verificou-se uma associação direta entre o IDH e a diferença de mortalidade atribuída à diabetes.

Diabetes mellitus; mortalidade; Peru. 